\title{
Forward hadron production at the LHC
}

\author{
Yoshitaka Itow $^{1,2, a}$ \\ 1 Solar-Terrestrial Environment Laboratory, Nagoya University \\ 2 Kobayashi-Maskawa Institute for the Origin of Particles and the Universe
}

\begin{abstract}
Observations of ultra high energy cosmic rays by extensive air showers rely on correct modeling of hadronic interaction in the forward region at very high energy. Recent new data from the LHC, especially for forward particle production, are important for a precise understanding of air shower development. In this article recent forward production data from p-p, $\mathrm{p}-\mathrm{Pb}$, and $\mathrm{Pb}-\mathrm{Pb}$ collisions at $\mathrm{LHC}$ are reviewed.
\end{abstract}

\section{Introduction}

The origin of ultra high energy cosmic rays (UHECR's) with energies reaching $10^{20} \mathrm{eV}$ is still enigmatic. Recent progress of observation by gigantic air shower experiments such as AUGER [1] and Telescope Array [2] reveal their energy spectrum, chemical composition and arrival directions by hybrid techniques with improved statistics. But yet no conclusive picture has been obtained. For example, determination of chemical composition and uncertainty of hadronic interactions always degenerate. Also possible excess in ground muon density recently reported by AUGER may hint a smoking gun of something new in hadronic interactions. These issues should be verified by accelerator data.

LHC can provide indispensable data set to understand hadronic interactions at such high energy. Proton-proton collisions at $\sqrt{s}=14 \mathrm{TeV}$ of $\mathrm{LHC}$ is equivalent to collisions of UHECRs with $10^{17} \mathrm{eV}$ in the laboratory frame. It is a good opportunity to serve various hadron interaction data at LHC to verify current UHECR analyses. There are several key observables to characterize hadron interactions relevant to air shower development. The first is inelastic cross section, which has been measured by Roman pot measurements such as TOTEM or ATLASALFA as well as by using minimum bias events. The second is production spectra of forward particles which carry most of the collision energy. The third is "inelasticity", which is defined as a fraction of the collision energy used to produce secondary particles. In addition, nuclear effects such as nuclear shadowing, secondary particle re-scattering, fermi motion, etc., are also important, since interactions in air showers are likely nucleon-Nitrogen collisions.

Forward produced particles, which are most relevant to air shower development, are produced from "diffractive" process where produced particles with small transverse momentum have a large rapidity gap. On the other hand, "non-diffractive" process produces a lot of particles at the central rapidity with smaller longitudinal momentum.

a e-mail: itow@stelab.nagoya-u.ac.jp
When a proton-proton collision happens, many QCD color strings are formed between projectile and target particles. These strings fragment and then form hadrons in the central rapidity region. On the other hand, many of the forward produced particles come from fragmentation of projectile or target particles. Particles from these two production mechanisms, diffractive and tail of nondiffractive productions, mix up in the forward rapidity region. In pp collisions at $\sqrt{s}=7 \mathrm{TeV}$, typically a half (20\%) of collisions is due to non-diffractive (diffractive) scatterings. The rest are elastic scattering events.

In the central rapidity, particle production can be well described by perturbative QCD processes. In the forward rapidity, theoretical modeling of these diffractive processes needs treatment of non-perturbative QCD at very high energy and small transverse momentum. PYTHIA, a hadronic interaction model which is commonly used in high energy physics experiments. It has been well tested by various accelerator data and well describe particle productions from perturbative QCD processes in the central rapidity region. Diffractive processes are also taken into account to accommodate the forward region. On the other hand, cosmic ray interaction models have been developed based on the Gribov-Regge theory where forward scattering amplitudes are naturally taken into account via multi-Pomeron exchange processes with connection to total cross section. So far SYBILL, DPMJET, QGSJET II, and EPOS are often used to simulate air showers. Some of the models are also available to simulate nuclear interactions.

Inputs from experimental data at LHC, especially for forward regions, have been utilized to verify and improve these interaction models. Four big experiments at the LHC, ATLAS, CMS, ALICE and LHCb, have various detectors covering the forward rapidity regions as well as the central rapidity regions. The CMS hadronic forward calorimeter (HF) is a steel/quartz-fiber calorimeter covering pseudorapidity $2.9<|\eta|<5.2$ [3]. The ATLAS forward calorimeters (Fcal) are liquid-Argon calorimeters with copper or tungsten converters covering $3.1<|\eta|$ $<4.9$ [4]. For the higher pseudorapidity region, the CMS

This is an Open Access article distributed under the terms of the Creative Commons Attribution License 4.0, which permits unrestricted use, distribution, and reproduction in any medium, provided the original work is properly cited. 


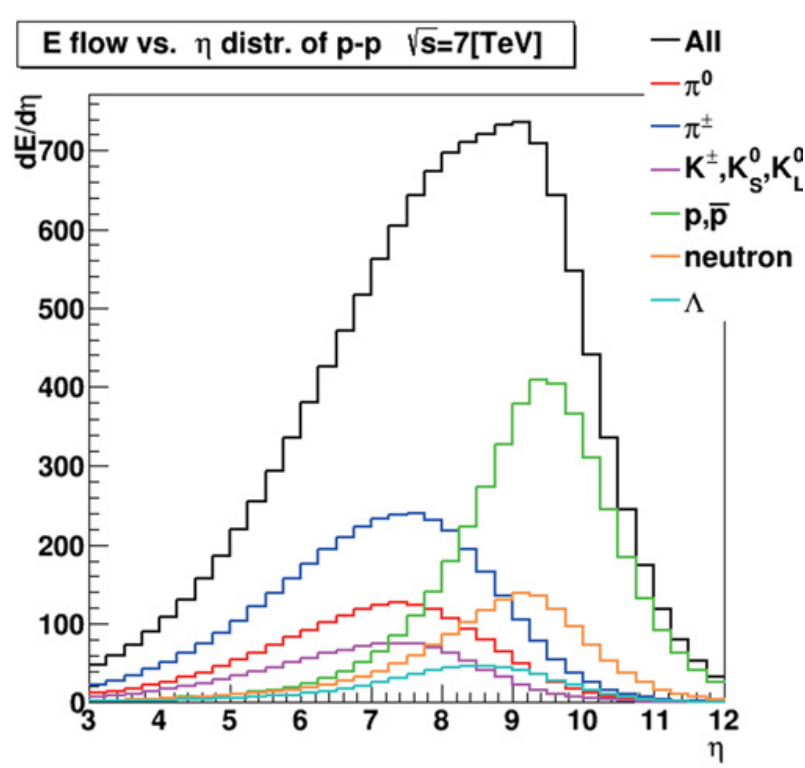

Figure 1. Energy flow as a function of pseudorapidity $\eta$ for various particles produced from $\sqrt{s}=7 \mathrm{TeV}$ pp collisions simulated by QGSJET II-04. (Figure courtesy of Dr. Nobuyuki Sakurai, KMI Nagoya University.)

CASTOR calorimeter, which is a Cherenkov detector using quartz plates interleaved with tungsten absorbers, locates at $z=-14.37 \mathrm{~m}$ from IP5 covering $-6.6<\eta<$ -5.2 [5]. ATLAS, CMS and ALICE have Zero Degree Calorimeters (ZDC), which are Cherenkov detectors using quartz fibers or quartz plates embedded in tungsten converters. They are located $140 \mathrm{~m}$ apart from IP's covering $|\eta|>8.3[3,4,6]$. Here only neutral particles reach after all the charged particles are swept away by the D1 magnets. In addition, two dedicated experiments, TOTEM and LHCf, have unique detectors to cover the very forward region. TOTEM has the Roman pots and the T1, T2 telescopes [7]. T1 locates $7.5 \mathrm{~m}$ to $10.5 \mathrm{~m}$ from IP5 covering $3.1<|\eta|<4.7$, and T2 locates $13.5 \mathrm{~m}$ apart from IP5 covering $5.3<|\eta|<6.5$. The LHCf has a pair of small tungsten sandwich calorimeters with four planes of position sensitive layers in front of ATLAS ZDC's at both sides of IP1 [8]. In this article, recent measurements of forward particle production by LHC experiments relevant to air shower development is discussed.

\section{Forward spectra, energy flow and multiplicity}

Figure 1 shows the energy flow from $\sqrt{s}=7 \mathrm{TeV}$ $\mathrm{pp}$ collisions as a function of pseudorapidity for various forward particles such as neutral pions, charged pions, Kaons, protons, neutrons, $\Lambda$ 's etc. predicted by QGSJET04. Here all the solid angles were integrated. The energy flow of mesons peaked at $\eta=7 \sim 8$, while those of protons and neutrons peaked at $\eta=9 \sim 10$. Therefore LHCf or ZDC can cover the peak of neutron energy flow, but can just cover the higher energy tail of the $\pi^{0}$ (or $\gamma$ ) energy flow. This coverage could be improved in the case of $\sqrt{s}=13 \mathrm{TeV}$ pp run as shown in Fig. 2. The minimum accessible pseudorapidity at LHCf/ZDC location is almost

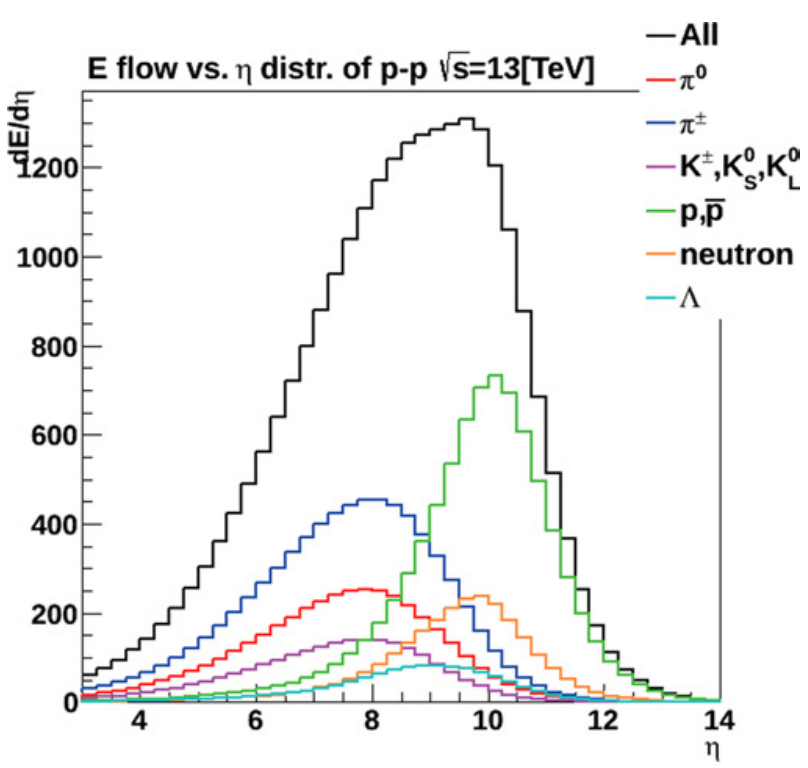

Figure 2. Energy flow as a function of pseudorapidity for various particles produced from $\sqrt{s}=13 \mathrm{TeV}$ pp collisions by QGSJET II-04. (Figure courtesy of Dr. Nobuyuki Sakurai, KMI Nagoya University.)

close to the pseudorapidity for the energy flow peak of mesons.

CMS reported the forward energy flow for minimum bias events from $\sqrt{s}=0.9$ and $7 \mathrm{TeV}$ pp collisions measured by HF [9]. The results of 5 bins $d E / d \eta$ in the pseudorapidity region $\eta=3.15$ to 4.19 were compared with various cosmic ray interaction models such as SIBYLL, QGSJET-01, QGSJET II-03 and EPOS1.99 and also with other high energy physics generators such as PYTHIA6 or PYTHIA8 tunes. It was pointed out that PYTHIA tunes without mutli-parton interactions could not produce enough energy flow in the forward region. Any cosmic ray interaction models include the effect of multiparton interactions and showed reasonable agreement with data without any tuning.

In the most forward region, LHCf provided the energy spectra for neutral particles produced at zero degree. The LHCf detector has the unique capability for discriminating between electro-magnetic showers and hadronic showers by using longitudinal shape of development. Thus forward energy flow can be measured separately for electromagnetic and hadronic components.

The energy spectra of single $\gamma$-rays were measured in $8.81<\eta<8.99$ and $10.94<\eta$ for $7 \mathrm{TeV}$ pp collisions [11]. They are compared with cosmic ray interaction models, DPMJET3.04, QGSJET II-03, SIBYLL2.1 and EPOS1.99 together with PYTHIA8.145. Whereas none of the models did not reproduce the energy spectra completely, EPOS1.99 and QGSJET II-03 reproduced relatively better than the other models. On the other hand, the data points lie just in the middle of the range of model difference, and is well bracketed by the lowest models and the largest models. This means current quoted size of systematic errors due to uncertainty in hadronic interactions deduced from the difference among the various existing interaction models would be adequate. 


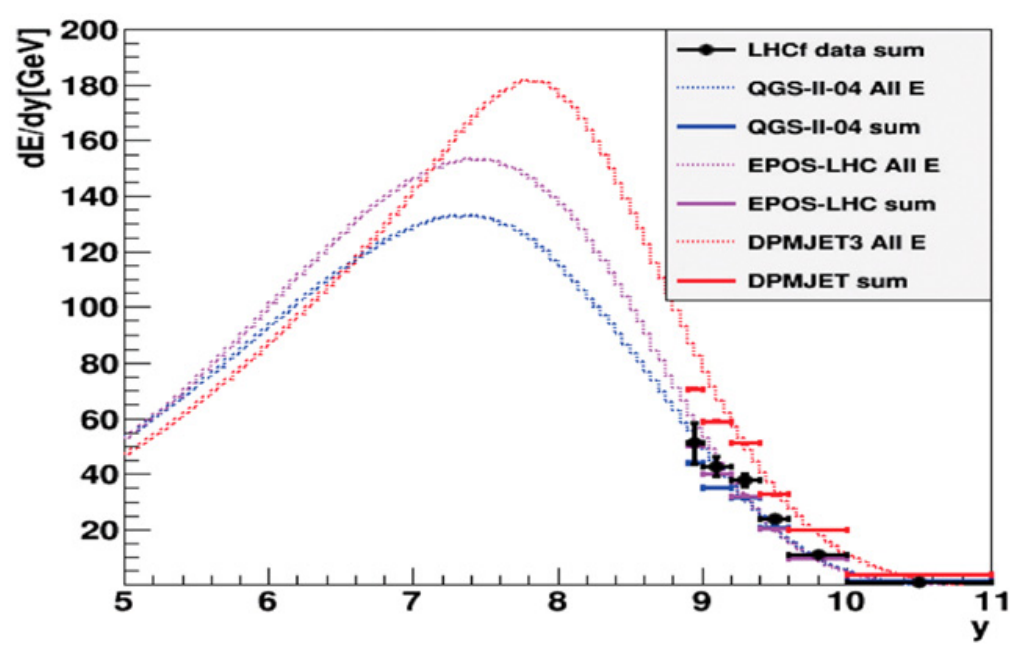

Figure 3. Energy flow as a function of rapidity $y$ for $\pi^{0} \mathrm{~s}$ from $\sqrt{s}=7 \mathrm{TeV}$ pp collisions simulated by QGSJET II-04 (blue dotted), EPOS-LHC (purple dotted), and DPMJET3 (red dotted). Also shown by solid histograms are the energy flow after the detector acceptance cut, which were simulated by three interaction models in six rapidity bins used in the LHCf $7 \mathrm{TeV} \mathrm{pp} \pi^{0}$ analysis [14]. The black crosses show the data energy flow obtained from the LHCf $\pi^{0}$ data. (Figure courtesy of Dr. Nobuyuki Sakurai, KMI Nagoya University.)

Similarly LHCf measured the energy spectra of single $\gamma$-rays in $8.81<\eta<8.99$ and in $10.94<\eta$ for $0.9 \mathrm{TeV}$ pp collisions [13]. Relative difference between the data and each model shows as similar behavior as that for the $7 \mathrm{TeV}$ pp sample. This suggests that good scaling of Feynman $X_{F}$, where $X_{F}=p_{z} / p_{\text {beam }}$, in $\mathrm{TeV}$ collisions, and the source of the discrepancy may be irrelevant to the collision energy. Indeed two Feynman $X_{F}$ distributions constructed from the energy spectra of $\gamma$-ray at the zero degree of $7 \mathrm{TeV}$ and $0.9 \mathrm{TeV}$ pp collisions are consistent with each other [12]. This collision energy dependence or Feynman $X_{F}$ scaling issue is important to extrapolate the forward results at $\mathrm{LHC}$ to much higher energy region accurately i.e. UHECR interactions at $10^{20} \mathrm{eV}$.

LHCf has good capability to reconstruct $\pi^{0}$ from 2 $\gamma$-rays. The $p_{T}$ distributions in six rapidity bins in $8.9<$ $y<11.0$ have been shown in [14]. The results shows that EPOS1.99 and QGSJET II-03 show almost consistent $p_{T}$ spectra with data, while DPMJET3 or PYTHIA show too hard spectra. Dominant fraction of single $\gamma$-rays detected by LHCf comes from $\pi^{0}$ decays. The single- $\gamma$ and $\pi^{0}$ samples cover the different area of the $\eta-p_{T}$ phase space. Thus two samples provide complemental information to understand all the production phase space of electromagnetic component. In order to cover entire region of phase space, reconstruction of higher energy $\pi^{0} \mathrm{~s}$ where two $\gamma$-rays from a $\pi^{0}$ decay incident at single calorimeter tower. A new analysis to reconstruct such higher energy $\pi^{0} \mathrm{~s}$ is now ongoing.

Figure 3 shows the energy flow carried by forward produced $\pi^{0} \mathrm{~s}$ as a function of rapidity $y$ for various interaction models. Also shown the LHCf data for six rapidity bins without acceptance correction together with the predicted energy flow in the same six rapidity bins after LHCf acceptance cuts were applied. The energy flow of $\pi^{0}$ from $\sqrt{s}=7 \mathrm{TeV}$ pp collisions peaked at $y \sim 8$. The rapidity region covered by LHCf is almost the tail of the energy flow peak where QGSJET II-04 and EPOS-LHC gave similar prediction. The measured values of energy flow were well reproduced by both of the model, while DPMJET3 overestimated by about $50 \%$.

LHCf has also reported the energy spectra of very forward neutron production in the three pseudorapidity ranges of $8.81<\eta<8.99,8.99<\eta<9.22$, and $\eta>10.76$ [15]. The obtained data were unfolded for $40 \%$ energy resolution, and compared with various cosmic ray interaction models. There are interesting difference between data and models. First, the observed production yields of very forward neutrons relative to those of very forward gamma rays show much larger than predicted by interaction models. Especially it was $2 \sim 3$ times larger for the most forward pseudorapidity $|\eta|>10.76$. The distributions of Feynman $X_{F}$ also show important information related to inelasticity. In the lower psuedorapidity regions such as $8.81<\eta<$ 8.99 and $8.99<\eta<9.22$, the $X_{F}$ distributions were reasonably reproduced by the models. The distribution generally increases as $X_{F}$ increases, and peaked around $X_{F} \sim 0.5$, then decreases towards $X_{F} \sim 1$. Here DPMJET3 showed nice agreement. On the other hand, the data spectrum showed the large bump shape, and it continuously increased toward $X_{F} \sim 1$ in the most forward pseudorapidity bin $\eta>10.76$. The bumpy shape might be reproduced by some of interaction models at larger $X_{F}$, but they were generally underestimated. Only $X_{F}$ from QGSJET II-03 models showed similar behavior, and continuously increased toward $X_{F}=1$. This typical feature of bump at large $X_{F}$ might be connected to the diffractive mass distribution in low mass diffraction events. The QGSJET II-03 model has a rapid increase of population at lower diffraction mass region, while other models assume flat diffraction mass distributions [16].

The $X_{F}$ distribution for neutrons produced at zero degree measured by ZDC in the RHIC PHENIX experiment for $\sqrt{s}=200 \mathrm{GeV}$ pp collision was reported together with those measured at ISR for $\sqrt{s}=30.6 \sim 62.7 \mathrm{GeV}$ pp collisions [18]. Interestingly the $X_{F}$ distribution from PHENIX data showed a similar bump peaked at $X_{F} \sim 0.8$, 


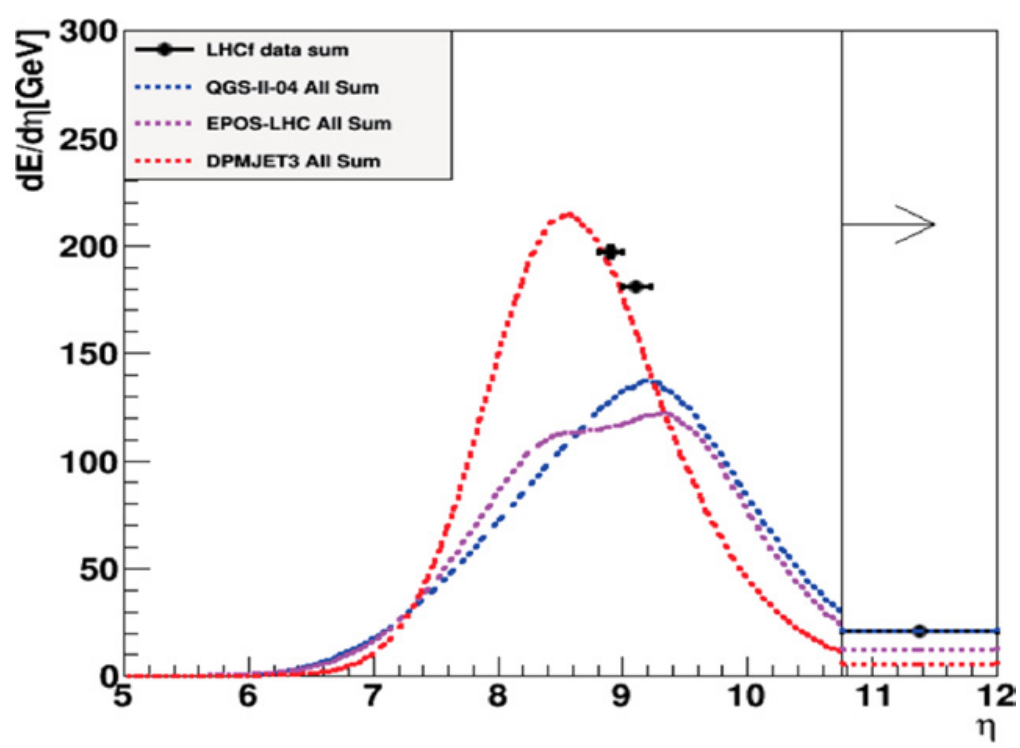

Figure 4. The energy flow as a function of pseudorapidity $\eta$ for various particles produced from $\sqrt{s}=7 \mathrm{TeV}$ pp collisions simulated by QGSJET II-04 (blue), EPOS-LHC (purple), and DPMJET3 (red). The black crosses show the energy flow in three psuedorapidity bins obtained from LHCf neutron data [15]. (Figure courtesy of Dr. Nobuyuki Sakurai, KMI Nagoya University.)

while it rapidly decreased to zero at $X_{F} \sim 1$. This feature was also consistent to those from ISR data at $\sqrt{s}=30.6 \sim$ 62.7 GeV. This $X_{F}$ behavior was also discussed regarding as the forward neutron productions from ep collisions at HERA [19]. In this work, neutron $X_{F}$ distributions in the same pseudorapidity ranges used in the LHCf analysis were deduced from the parametrization based on HERA measurement and compared to those from LHCf data. In the lower pseudorapidity ranges $8.81<\eta<8.99$ and $8.99<\eta<9.22$, the $X_{F}$ distributions deduced from HERA measurement reasonably reproduced both the shape and the height of LHCf $X_{F}$ data. However, in the most forward bin $\eta>10.76$, HERA parametrization predicted the $X_{F}$ peaked at $\sim 0.8$ and decreased to zero at $X_{F}>0.8$, which was similar to the PHENIX and the ISR results. There may be tension with that from LHCf at $X_{F} \sim 1$. The HERA forward neutron production data was basically well reproduced by the one-pion exchange model but it still predicted a rapid decrease at $X_{F} \sim 1$. Possible discrepancy on zero degree neutron production at $X_{F} \sim 1$ between LHCf $7 \mathrm{TeV}$ pp data and other data may provide some new hints.

Figure 4 shows the energy flow as a function of pseudorapidity for various interaction models together with those measured by LHCf. Despite the large discrepancy observed in the data, neutron energy flow in the most forward pseudorapidity did not show so large impact, because geometrical acceptance is anyway small. The neutron energy flow peaked at $\eta \sim 8.5$ where two lower psuedorapidity bins of LHCf data just covered. Here DPMJET3 well reproduced neutron energy flow at the peak region, while QGSJET II-04 or EPOS-LHC underestimated by about $30 \%$.

Between the pseudorapidity region covered by CMS $\mathrm{HF}$ and that by LHCf, the CMS CASTOR calorimeter covers unique psuedorapidity region $-6.6<\eta<-5.2$, where the energy flow rapidly rises up toward the peak at $\eta \sim 7.5$. So far CASTOR has provided relative comparison of forward energy density between hard events and

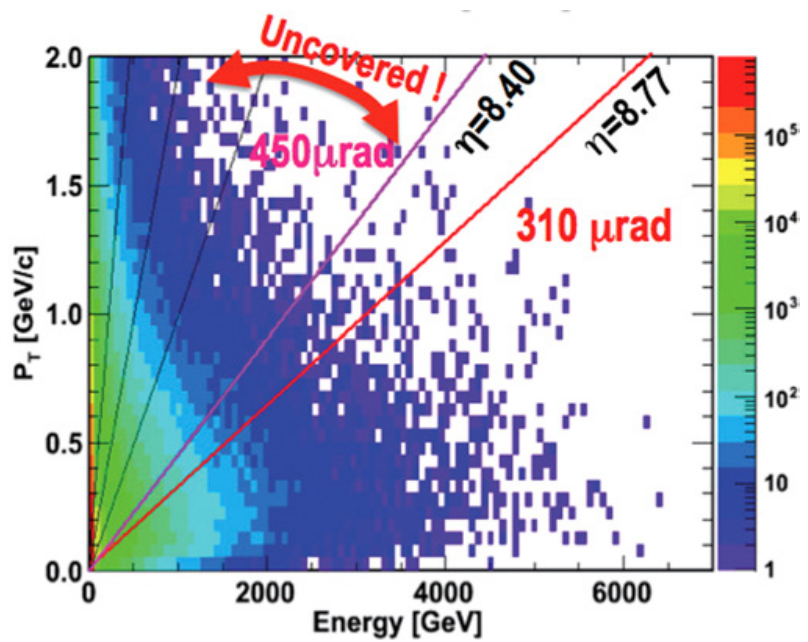

Figure 5. The 2-dimensional histograms of energy vs $p_{T}$ for $\gamma$ 's from $\sqrt{s}=13 \mathrm{TeV}$ pp collisions simulated by QGSJET II-03. The lines indicate corresponding pseudorapidity regions.

inclusive events for $\sqrt{s}=0.9 \mathrm{TeV}, 2.76 \mathrm{TeV}$ and $7 \mathrm{TeV}$ pp collisions [17]. Here "minium-bias" inclusive events were selected by requiring one primary vertex and a hit at both HF and CASTOR with certain energy thresholds (a few GeV). Track-jet events were used to identify hard-scattered events. The hard events were selected by requiring a leading track-jet with $p_{T}>1 \mathrm{GeV} / c$ in $\left|\eta^{\text {jet }}\right|<2$. After correcting all the selection efficiency and reconstruction bias, the energy flow ratios between hard events and inclusive events per pseudorapidity bin, $\left(d E^{\text {hard }} / d \eta\right) /\left(d E^{\text {incl }} / d \eta\right)$, were calculated as functions of leading charged jet $p_{T}$ for $\mathrm{pp}$ collisions at three different collision energies, $0.9 \mathrm{TeV}, 2.76 \mathrm{TeV}$ and $7 \mathrm{TeV}$. As shown in Figs. 4 and 5 in Ref [17], the hard-inclusive energy flow ratio for $\sqrt{s}=7 \mathrm{TeV}$ collisions increased as $p_{T}$ of leading charged jet increased. This $p_{T}$ dependence of the energy flow ratio became flat for $\sqrt{s}=2.76 \mathrm{TeV}$, while it decreased as jet $p_{T}$ increased in the case of $\sqrt{s}=0.9 \mathrm{TeV}$. 
These features could be interpreted as a contribution from multi-parton interactions to particle production is essentially large at large collision energy as $\sqrt{s}=7 \mathrm{TeV}$. The track-jet $p_{T}$ in the central rapidity is a good indicator of hard-scale of parton-parton collisions. Larger (smaller) track-jet $p_{T}$ values indicate that more central (peripheral) collisions take place, resulting more (less) forward energy flow for $\sqrt{s}=7 \mathrm{TeV}$. On the contrary, in $\sqrt{s}=0.9 \mathrm{TeV}$, energy flow in the forward pseudorapidity comes largely from inclusive events, and thus the events with larger $p_{T}$ of central track-jets could not provide energy for the forward region. The relative dependence of energy flow on three collision energies were also obtained for the inclusive events and the hard jet events where jet $p_{T}>10 \mathrm{GeV} / c$ was required. A rapid increase of energy flow with increase of collision energy from $2.76 \mathrm{TeV}$ to $7 \mathrm{TeV}$ was observed. These features are more or less reproduced by cosmic ray interaction models, while interaction models which do not consider multi-parton interactions significantly underestimate the energy flow in $7 \mathrm{TeV}$. This also indicates an important contribution from multi-parton interactions to forward particle production in multi $\mathrm{TeV}$ pp collisions.

Charged multiplicity per pseudorapidity bin $d N_{c h} / d|\eta|$ in $\sqrt{s}=8 \mathrm{TeV}$ pp collisions have been obtained by collaborative analysis of CMS and TOTEM [20]. Here the CMS detector and the TOTEM T2 telescopes measured $|\eta|<2.2$ and $5.3<|\eta|<6.4$, respectively. By combined data analysis, direct comparison of forward multiplicity to central multiplicity for common data sample is now available. Data were collected by the minimum-bias trigger provided by the two TOTEM T2 telescopes, when at least one track candidate in either side of $\mathrm{T} 2$ telescope. The results of $d N_{c h} / d|\eta|$ were obtained for three different data samples, inclusive $\mathrm{pp}$, NSD-enhanced pp, and SD-enhanced pp samples. In the inclusive and the NSD-enhanced pp samples, the results both in central and forward regions were well described by QGSJET II-04. However in the SD-enhanced pp sample QGSJET II-04 underestimated by about 20-30\%. For the entire pseudorapidity region, EPOS-LHC overestimated by about $10-20 \%$ in the inclusive and the NSD-enhanced samples, and underestimated by about $10 \%$ in the SDenhanced sample. They are still almost within the quoted experimental errors.

\section{Nuclear effects in $\mathrm{PbPb}$ and $\mathrm{pPb}$ collisions}

Since cosmic rays actually interact with nitrogen or other nuclei, nuclear effects in secondary particle productions should be correctly taken into account. There have been many studies in central rapidity regions of heavy ion (AA) collisions regarding as Quark Gluon Plasma (QGP) and also proton-ion ( $\mathrm{pA}$ ) or deuteron-ion ( $\mathrm{dA}$ ) collisions as reference data for QGP study. Yet detailed studies in forward particle production in AA or pA have not been made. LHC provided $\mathrm{PbPb}$ collisions at $\sqrt{s_{N N}}=2.76 \mathrm{TeV}$ and $\mathrm{pPb}$ collisions at $\sqrt{s_{N N}}=5.02 \mathrm{TeV}$. These data are also important for a precise understanding of air showers.

The energy flow per pseudorapidity for $\sqrt{s_{N N}}=$ 2.76 TeV $\mathrm{PbPb}$ collisions was measured by CMS together with the extended forward region $-6.6<\eta<-5.2$ covered by CASTER [21]. So far the absolute energy scale of CASTOR was calibrated by the energy flow measured for $7 \mathrm{TeV}$ pp collisions by HF which was extrapolated to the CASTOR pseudorapidity region by using various interaction models. With this method, the current understanding of the CASTOR energy scale has about $25 \%$ uncertainty. Minimum bias inelastic events were selected by requiring either HF or BSC have signals on both sides of IP. Additional offline cuts were applied to eliminate background due to ultra peripheral collisions or beam halo interactions. Energy density and transverse energy density, $d E / d \eta$ and $d E_{T} / d \eta$, were measured in the pseudorapidity range $-6.6<\eta<5.2$ for different centralities. The results in the wide range of pseudorapidity were compared with various interaction models such as HYDJET and AMPT as well as cosmic ray interaction models EPOS-LHC and QGSJET II-03. For central collision events EPOS-LHC showed better agreement, while QGSJET II-03 worked better for peripheral events.

The effect of cold nuclear matter in proton-nucleus collisions can be expressed as the difference in particle productions between proton-nucleus collisions and protonproton collisions scaled by number of binary collision $\left\langle N_{\text {coll }}\right\rangle$ derived from the Glauber model calculation. A nuclear modification factor in proton-nucleus collisions $R_{\mathrm{pA}}$ can be;

$$
R_{\mathrm{pA}}=\frac{\sigma_{\text {inel }}^{\mathrm{pp}}}{\left\langle N_{\text {coll }}\right\rangle \sigma_{\text {inel }}^{\mathrm{pA}}} \frac{E d^{3} \sigma^{\mathrm{pA}} / d p^{3}}{E d^{3} \sigma^{\mathrm{pp}} / d p^{3}},
$$

where $\sigma_{\text {inel }}^{\mathrm{pp}}$ and $\sigma_{\text {inel }}^{\mathrm{pA}}$ are inelastic cross sections for pp and $\mathrm{pA}$ collisions, respectively. In the case of $\sqrt{s_{N N}}=$ $5.02 \mathrm{TeV} \mathrm{pPb}$ collisions at LHC, $\left\langle N_{\text {coll }}\right\rangle=6.9 \pm 0.7$ was chosen for hard production.

Nuclear modification factors have been studied at RHIC for $\sqrt{s_{N N}}=200 \mathrm{GeV} \mathrm{dAu}$ collisions with comparison to $200 \mathrm{GeV}$ pp collision data by using Glauber model calculation. The STAR experiment observed suppression of $\pi^{0}$ production $\left(25<E_{\pi}<55\right)$ in the forward pseudorapidity $3.0<\eta<4.2$ corresponding to $p_{T}=1-2 \mathrm{GeV} / c$ region [22]. Observed $R_{d A u}$ was about 0.3 , which was bigger than expectation from shadowing effects indicating possible gluon saturation effect. Also RHIC BRAHMS measured $R_{d A u}$ for charged hadron production at $\eta=0,1,2.2$ and 3.2 down to $p_{T}$ $<1 \mathrm{GeV} / c$ region [23]. The result possibly suggests suppression became larger for higher pseudorapidity and larger for central collisions. These results indicate strong suppression of forward particle production due to nuclear effects in high energy proton-nucleus collisions. This suppression effect might be larger for the very forward region because of the large parton density resulting in gluon saturation [24].

Nuclear modification factors $R_{\mathrm{pPb}}$ and $R_{\mathrm{PbPb}}$ for various secondary particle production were studied in $\sqrt{s_{N N}}=5.02 \mathrm{TeV} \mathrm{pPb}$ and $\sqrt{s_{N N}}=2.76 \mathrm{TeV} \mathrm{PbPb}$ collisions at LHC, respecitively. ALICE reported $R_{\mathrm{pPb}}$ and $R_{\mathrm{PbPb}}$ for charged particle production in the central rapidities $\left|\eta_{\mathrm{CMS}}\right|<0.3$ and $|\eta|<0.8$, respectively [25]. They observed strong suppression in $R_{\mathrm{PbPb}}$ in the entire 
range of $p_{T}<20 \mathrm{GeV} / c$, while $R_{\mathrm{pPb}}$ remained unity above $p_{T}=2 \mathrm{GeV} / c$. ALICE also reported $R_{\mathrm{pPb}}$ for forward heavy quarkonium productions such as $\mathrm{J} / \psi$ and $\psi(2 \mathrm{~S})$ [26].

The LHCf experiment reported $R_{\mathrm{pPb}}$ for very forward $\pi^{0}$ production measured by the LHCf Arm 2 detector at proton remnant side. [27]. The analysis methods were almost similar to that for $7 \mathrm{TeV} \pi^{0}$ analysis. In $\mathrm{pPb}$ collisions at zero degree, half of the $\pi^{0}$ s coming from $\Delta$ resonance of beam protons were due to ultra peripheral collision with $\mathrm{Pb}$ nuclei. They were subtracted from the data sample, and $R_{\mathrm{pPb}}$ ratios were calculated for six rapidity bins in the range of $-11.0<y<-8.9$. Here $\left\langle N_{\text {coll }}\right\rangle=6.9$ as same as used for the previous ALICE analysis was used for $R_{\mathrm{pPb}}$ calculation, while observed $p_{T}$ range was less than several hundred $\mathrm{MeV} / c$. The obtained $R_{\mathrm{pPb}}$ ratios were about 0.1 for all rapidity bins. The results were compared to DPMJET3, QGSJET II-03, and EPOS 1.99 , and these models reasonably reproduced the data $R_{\mathrm{pPb}}$.

\section{Future prospects}

Many continuous efforts have been made to understand cosmic ray interactions, especially, for forward particle productions at the LHC. There have been some discussions about idea for possible future measurements at LHC.

First, a proton-light ion collision run will provide important direct calibration of nuclear effect in protonnitrogen interactions in the air shower. An oxygen beam is a good candidate for a possible light ion beam in LHC, since oxygen gas is commonly used for support gas in current ion source operation. For example, energy flow in the forward region of oxygen-proton collisions at $\sqrt{s}=$ 4.9 TeV shows about $25 \%$ difference between QGSJET II04 and EPOS-LHC [28]. This feature may not be resolved by approximation from the $\mathrm{pPb}$ collision data for protonlight ion collisions. Direct measurements in a protonOxygen run would be conclusive.

The other issue would be an extension of rapidity coverage to the current inaccessible region, So far CMS with CASTOR covers pseudorapidity range $-6.6<\eta<$ 5.2, and LHCf covers $|\eta|>8.40$ with $140 \mu \mathrm{rad}$ beam crossing angle. The inaccessible pseudrorapidity $-8.4<$ $\eta<-6.6$ is an important and interesting region in the case of $\sqrt{s}=13 \mathrm{TeV}$ pp collisions where energy flow of $\pi$ 's has a peak, and almost half of the electro-magnetic energy flow is deposited as shown in Fig. 2. Also by covering this pseudorapidity region, we can cover the higher $p_{T}$ region for $p_{T}>1 \mathrm{GeV} / c$ as shown in Fig. 5. In this $p_{T}$ region, we could observe an interesting transition from diffraction to non-diffraction, or from non-perturbative QCD regime to perturbative QCD regime. The accessible rapidity and $p_{T}$ range are limited by the beam pipe shadow. For example pseudorapidity acceptance of CASTOR is limited by the beam pipe diameter in the IP region, and LHCf/ZDC is also limited by the vertical aperture of beam pipe at the exit of D1 magnet. Some new idea is indispensable to access the missing pseudorapidity region, which may have a key to understand cosmic ray interactions at ultra high energy.
The author would like to thank Nobuyuki Sakurai and Colin Baus for providing useful plots and slides. This work was supported by Gran-in-Aids for Scientific Research by MEXT of Japan.

\section{References}

[1] A. Aab, et al. (The Pierre Auger Collaboration), arXiv:1307. 5059 (2013)

[2] H. Sagawa et al., (The Telescople Array Collaboration), Braz. J. Phys. 44, 589 (2014)

[3] S. Chatrchyan et al. (The CMS Collaboration), JINST 3, S08004 (2008)

[4] S. Chatrchyan et al. (The ATLAS Collaboration), JINST 3, S08003 (2008)

[5] V. Andree et al., Eur. Phys. J. C67, 67 (2010)

[6] G. Dellacasa et al. (The ALICE Collaboration), CERN-LHCC-99-05 (1999)

[7] G. Anelli et al. (The TOTEM Collaboration), JINST 3, S08007 (2008)

[8] O. Adriani et al. (The LHCf Collaboration), JINST 3, S08006 (2008)

[9] S. Chatrchyan et al. (The CMS Collaboration), JHEP 11, 148 (2011), Erratum-ibid, JHEP 02, 055 (2012)

[10] S. Chatrchyan et al. (The CMS Collaboration), JHEP 04, 072 (2013)

[11] O. Adriani et al. (The LHCf Collaboration), Phys. Lett. B703, 128 (2011)

[12] K. Taki, PhD Thesis, Nagoya University, 78 pages (2012)

[13] O. Adriani et al. (The LHCf Collaboration), Phys. Lett. B715, 298 (2012)

[14] O. Adriani et al. (The LHCf Collaboration), Phys. Rev. D86, 092001 (2012)

[15] K. Kawade, PhD Thesis, Nagoya University, 124 pages (2014)

[16] S. Ostapchenko, Talk at HESZ2013 Workshop, Nagoya, Japan, 4th, March (2013)

[17] S. Chatrchyan et al. (The CMS Collaboration), JHEP 04, 072 (2013)

[18] A. Adare et al. (The PHENIX Collaboration), Phys. Rev. 88, 032006 (2013)

[19] Y.Yamazaki, Talk at LOWX2014 Workshop, Kyoto, Japan, 18th, June, 2014

[20] S. Chatrchyan et al. (The CMS and TOTEM Collaboration), Eur. Phys. J. C74 3053 (2014)

[21] S. Chatrchyan et al. (The CMS Collaboration), CMSPAS-HIN-12-006 (2012)

[22] J. Adams et al. (The STAR Collaboration), Phys. Rev. Lett. 97, 152302 (2006)

[23] I. Arsene et al. (The BRAHMS Collaboration), Phys. Rev. Lett. 93, 242303 (2004)

[24] Dumitru, Gerland and Strikman, Phys. Rev. Lett. 90, 092301 (2003)

[25] B. Abelev et al. (The ALICE Collaboration), Phys. Rev. Lett. 110, 082302 (2013)

[26] B. Abelev et al. (The ALICE Collaboration), arXiv:1405.3796 (2014)

[27] O. Adriani et al. (The LHCf Collaboration), Phys. Rev. C89, 065209 (2014)

[28] T. Pierog, Talk at "QCD and Forward Physics at the LHC”, Trento, Italy 17th, April (2014) 